embolization with complete occlusion of the aneurysm on 6month follow up catheter angiography. She was subsequent lost to follow-up, but represented 12 years later after undergoing treatment for a squamous cell carcinoma of the palate with interval re-rupture and enlargement of her vertebrobasilar junction aneurysm to $12 \mathrm{~mm}$ in diameter. She underwent successful stent-assisted coiling with complete occlusion of the aneurysm. Two years later her aneurysm re-ruptured a third time after further enlarging to $13 \mathrm{~mm}$ and she underwent repeat coil embolization. Given progressive aneurysm enlargement and re-rupture despite multiple successful embolization procedures, she underwent vascular imaging of her chest revealing previously unknown complete occlusion of the right subclavian artery origin resulting in asymptomatic right-sided subclavian steal physiology. As her otherwise asymptomatic right subclavian steal physiology was likely a significant contributor to multiple recurrences of her vertebrobasilar junction aneurysm, she underwent surgical bypass of her right subclavian origin occlusion.

Conclusion Vertebrobasilar junction aneurysms are uncommon, but occasionally associated with subclavian steal physiology and can recur despite successful endovascular treatment. Although asymptomatic subclavian steal physiology is usually managed conservatively, associated vertebrobasilar junction aneurysms may represent an indication for treatment of the subclavian artery occlusion to decrease the risk of aneurysm recurrence.

Disclosures O. Stechishin: None. J. Corona: None. T. Binyamin: None. R. Novakovic: None. J. Barr: None. J. White: None. R. de Oliveira Sillero: None. B. Welch: None. L. Pride: None.

\section{E-032 RECONSTITUTION OF THE INTERNAL CAROTID ARTERY BY THE VASA VASORUM IS ASSOCIATED WITH AN APLASTIC OR HYPOPLASTIC CIRCULUS ARTERIOSUS}

${ }^{1} \mathrm{~V}$ Mayercik*, ${ }^{2} \mathrm{M}$ Marks, ${ }^{2} \mathrm{R}$ Dodd, ${ }^{2} \mathrm{~J}$ Heit, ${ }^{2} \mathrm{H}$ Do, ${ }^{2} \mathrm{~N}$ Telischak. ${ }^{1}$ Stanford University, Menlo Park, $C A ;{ }^{2}$ Stanford University, Stanford, $C A$

\subsection{6/neurintsurg-2021-SNIS.128}

Introduction The vasa vasorum are small vessels in the adventitial and medial layers of larger vessels which nourish their walls. When large vessels are occluded, the vasa vasorum may reconstitute them. We hypothesize that their hypertrophy is associated with a hypoplastic or aplastic circulus arteriosus which may be unable to meet the ischemic demands of the tissue perfused by the occluded vessel.
Materials and Methods We conducted a retrospective cohort study of patients with occlusion of the internal carotid artery and reconstitution of the vessel by vasa vasorum as confirmed by cerebral angiography. An electronic medical record was queried for patient demographics. The presence and caliber of an anterior communicating artery (AComm)and a posterior communicating artery (PComm) on the ipsilateral side of the lesion were measured by two experienced neurointerventionalists.

Results We reported 11 cases in 11 patients. Patients were predominantly female $(n=7)$ and older (mean age 63.7 years, SD 15.6 years). $100 \%$ of patients had either an aplastic or hypoplastic circulus arteriosus. $81.8 \%$ of patients had either an aplastic AComm or ipsilateral PComm. When these vessels were present, $63.6 \%$ were hypoplastic with a mean diameter of $0.85 \mathrm{~mm}$ (SD $\pm 0.34 \mathrm{~mm}$ ) and $0.82 \mathrm{~mm}$ (SD \pm 0.22 $\mathrm{mm})$, respectively.

Conclusion Reconstitution of an occluded internal carotid artery by the vasa vasorum is associated with an aplastic or hypoplastic circulus arteriosus, which may fail to compensate for the ischemic demand of the tissue initially perfused by the occluded vessel.

Disclosures V. Mayercik: None. M. Marks: None. R. Dodd: None. J. Heit: None. H. Do: None. N. Telischak: None.

\section{E-033 PROCEDURAL OUTCOME DEPENDING ARTERIAL DIAMETER IN THE ACUTE M2 OCCLUSIVE ISCHEMIC STROKE PATIENTS TREATED WITH ENDOVASCULAR THROMBECTOMY}

1J Kim*, ${ }^{1} \mathrm{~K}$ Lee, ${ }^{1} \mathrm{~J}$ Kang, ${ }^{1} \mathrm{~B}$ Kim, ${ }^{1} \mathrm{M}$ Han, ${ }^{1} \mathrm{H}$ Bae, ${ }^{2} \mathrm{~S}$ Baik, ${ }^{2} \mathrm{~L}$ Sunwoo, ${ }^{2} \mathrm{~K}$ Kim, ${ }^{2} \mathrm{C}$ Jung ${ }^{1}$ Department of Neurology, Seoul National University Bundang Hospital, Seongnam, Korea, Republic of; ${ }^{2}$ Department of Radiology, Seoul National University Bundang Hospital, Seongnam, Korea, Republic of

\subsection{6/neurintsurg-2021-SNIS.129}

Background Several reports showed that clinical outcome was differed by the occluded location of M2 segment of middle cerebral artery in the endovascular thrombectomy (EVT). However, there is no report about the association between arterial diameter and outcomes. We aimed to evaluate the relationship between arterial diameter of acute ischemic stroke patients treated with EVT for M2 occlusion and the outcomes.

Methods Using a prospective single center endovascular thrombectomy registry, we identified acute M2 occlusion patients who treated with EVT from 2011 to 2016. M2 diameter was measured at the proximal segment of occlusion

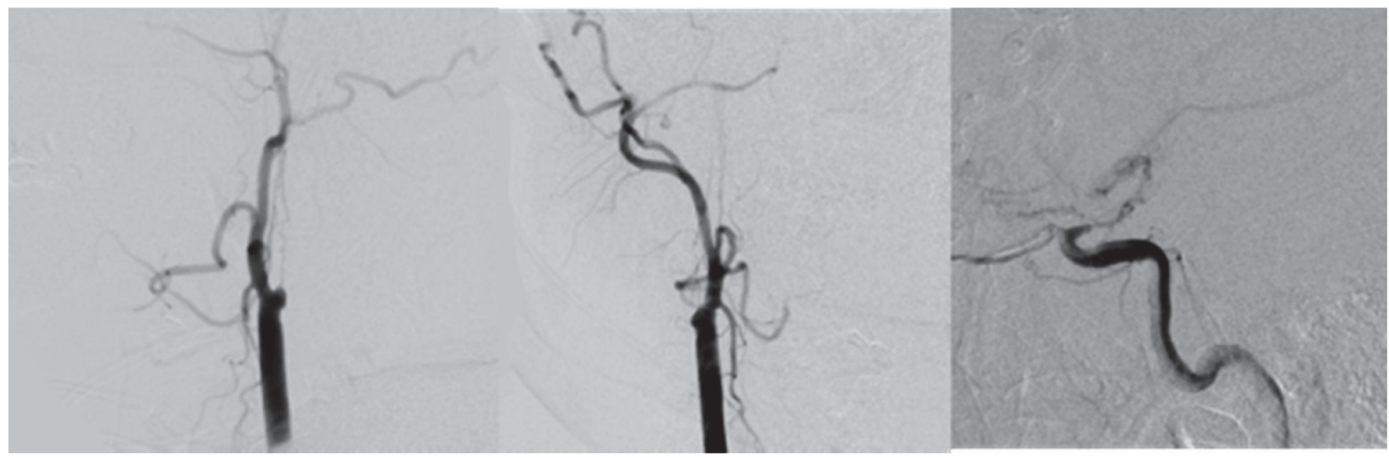

